\title{
DESEMPENHO DE LISÍMETRO DE PESAGEM HIDRÁULICA DE BAIXO CUSTO NO SEMI-ÁRIDO NORDESTINO ${ }^{1}$
}

\author{
FRANCISCO X. DOS SANTOS ${ }^{2}$, JOSÉ J. V. RODRIGUES ${ }^{3}$, \\ ABELARDO A. DE A. MONTENEGRO ${ }^{4}$, RONALDO F. DE MOURA ${ }^{5}$
}

\begin{abstract}
RESUMO: Medidas diretas de evapotranspiração de referência são importantes na avaliação e na calibração de métodos indiretos. Avaliou-se o desempenho de lisímetro de pesagem hidráulica de baixo custo, visando à determinação direta da evapotranspiração de referência (ETo), em área de assentamento rural no Semi-Árido nordestino. O lisímetro é de concepção simplificada, com travesseiros hidráulicos confeccionados com mangueira flexível de butil e revestida em náilon, interligados a um sistema manométrico com mercúrio e água. O equilíbrio do lisímetro foi estável, sendo o maior erro médio posicional de 3,93\%. As calibrações geraram coeficientes de correlação superiores a 0,9993 e resoluções de leituras entre 0,095 e 0,134 mm. As leituras lisimétricas foram comparadas com estimativas da ETo obtidas, utilizando-se dos métodos de Penman-Monteith, Blaney-Criddle-FAO, Jensen-Haise e do Tanque Classe A-FAO. Adicionalmente, avaliou-se o desempenho de um tanque evaporimétrico simplificado, construído a partir de material reciclado, o qual vem sendo utilizado no manejo da irrigação em áreas de agricultura familiar. Todos os métodos analisados apresentaram bom desempenho, com medidas bem correlacionadas às do lisímetro, para o período estudado.
\end{abstract}

PALAVRAS-CHAVE: lisímetro de pesagem, Penman-Monteith, necessidade hídrica, evapotranspiração.

\section{LOW COST HYDRAULIC WEIGHING LYSIMETER PERFORMANCE IN THE SEMI ARID NORTHEAST, BRAZIL}

\begin{abstract}
Direct evapotranspiration measurements are important to the calibration of indirect methods. The performance of a low cost hydraulic weighing lysimeter was evaluated, aiming to estimate the reference evapotranspiration (ETo) in a settlement area in the Semi Arid Brazilian northeast. The lysimeter presents a simplified design, with loading cells of butyl internally covered with nylon, connected to a manometric system with mercury and water. The lysimeter equilibrium was stable, with the largest positional average error of $3.93 \%$. Calibrations produced correlation coefficients higher than 0.9993, and reading resolutions between 0.095 and $0.134 \mathrm{~mm}$. Lysimeter readings were compared to ETo estimate Penman-Monteith, Blaney-Criddle FAO, Jensen-Haise, and Class A methods. Additionally, the performance of a simplified evaporimetric tank was evaluated, built with recycled material, which has been used for irrigation management in communal areas. All the considered methods produced good results, well correlated to the readings from the lysimeter, for the studied period.
\end{abstract}

KEYWORDS: weighing lysimeter, Penman-Monteith, water requirement, evapotranspiration.

\footnotetext{
${ }^{1}$ Extraído da dissertação de mestrado do primeiro autor.

${ }^{2}$ Eng $^{\mathrm{o}}$ Agrônomo, Doutorando, Programa de Pós-Graduação em Agronomia - Ciência do Solo, UFRPE, Recife - PE, Fone: (0XX81) 3320.6220, franciscoxaviersantos@hotmail.com

${ }^{3}$ Eng $^{\mathrm{o}}$ Agrônomo, Prof. Adjunto, Departamento de Agronomia, UFRPE, Recife - PE.

${ }^{4}$ Eng ${ }^{\mathrm{O}}$ Civil, Prof. Adjunto, Departamento de Tecnologia Rural, UFRPE, Recife - PE.

${ }^{5}$ Eng ${ }^{0}$ Agrônomo, Prof Adjunto, Departamento de Tecnologia Rural, UFRPE, Recife - PE.

Recebido pelo Conselho Editorial em: 30-10-2007
}

Aprovado pelo Conselho Editorial em: 19-2-2008 


\section{INTRODUÇÃO}

A evapotranspiração é um processo natural de perda de água do solo e da planta para a atmosfera, constituindo-se em parâmetro de grande relevância para o manejo agrícola, particularmente em regiões onde predominam elevados déficits hídricos anuais, como, por exemplo, o Semi-Árido nordestino. Nessas áreas, a irrigação, aliada à aquisição de dados confiáveis de evapotranspiração, torna-se essencial.

Os métodos indiretos para a estimativa da evapotranspiração constituem-se nos modelos agrometeorológicos e em tanques de evaporação (MENDONÇA et al., 2003). No Brasil, principalmente nas regiões Norte e Nordeste, os valores de evapotranspiração de referência utilizados no manejo de irrigação são, muitas vezes, obtidos por meio de modelos empíricos, como as equações de Blaney-Criddle e de Hargreaves, que, pela simplicidade, estão entre as mais utilizadas (MIRANDA et al., 1999). Entretanto, a confiabilidade desses modelos nem sempre é satisfatória, particularmente no Semi-Árido brasileiro, o que implica necessidade de testá-los em condições locais, diferentes daquelas em que foram desenvolvidos (MENDONÇA et al., 2003). Dentre os métodos diretos para a determinação da evapotranspiração, está a lisimetria, particularmente a de pesagem, procedimento mais preciso para avaliar a evapotranspiração (ABOUKHALED et al., 1982; MENDONÇA et al., 2003), entretanto usualmente inacessível à agricultura familiar.

SCHNEIDER et al. (1998) relatam que o uso de lisímetros convencionais de pesagem é limitado pelo alto custo da mão-de-obra especializada na construção e na operação, bem como pelo tamanho, pelos equipamentos especiais e materiais utilizados. LUNARDI et al. (1999) e MENDONÇA et al. (2003) ressaltam a importância da utilização de equipamentos simplificados que contemplem baixo custo, fácil operação e apresentem adequada precisão. SILVA (2005) verificou que os lisímetros simplificados podem apresentar boa precisão em escala diária, com custo de construção baixo, comparado aos construídos com células de carga eletrônica, por exemplo. Os lisímetros de pesagem hidráulica têm sido difundidos e aperfeiçoados, como se constata nos trabalhos de FREITAS (1994), COSTA (1999) e SILVA et al. (2003).

Neste contexto, teve-se o objetivo de avaliar o desempenho de lisímetro de pesagem hidráulica de baixo custo para a estimativa da evapotranspiração de referência, correlacionando-a àquela obtida a partir de modelos agrometeorológicos e de tanques evaporimétricos, em área de clima Semi-Árido do Brasil.

\section{MATERIAL E MÉTODOS}

O estudo foi realizado em área de assentamento rural da Fazenda Nossa Senhora do Rosário, onde se desenvolve agricultura familiar irrigada, na região Agreste de Pernambuco, nas coordenadas geográficas $08^{\circ} 24^{\prime} 11^{\prime \prime}$ sul e $37^{\circ} 48^{\prime} 54^{\prime \prime}$ oeste. O clima local é classificado, segundo Köeppen, como BShw', semi-árido quente, caatinga hiperxerófila, com temperatura média anual do ar em torno de $27^{\circ} \mathrm{C}$ e precipitação média anual de $600 \mathrm{~mm}$. A umidade relativa média anual do ar é de $73 \%$, e a velocidade média do vento é de $2,5 \mathrm{~m} \mathrm{~s}^{-1}$ (CISAGRO, 1990).

Durante o período experimental, de novembro de 2003 a janeiro de 2004, foram registrados, em estação automática, velocidade média do vento a $2 \mathrm{~m}$ de $0,80 \mathrm{~m} \mathrm{~s}^{-1}$, temperatura média diária do ar de $25^{\circ} \mathrm{C}$, umidade relativa do ar de $60 \%$, radiação solar média de $22,40 \mathrm{MJ} \mathrm{m}^{2}$ dia $^{-1}$ e total pluviométrico de $181,87 \mathrm{~mm}$. Os elementos meteorológicos do período experimental foram medidos em estação automática (Campbell Scientific - ET 106).

O tanque do lisímetro consistiu em uma caixa de fibra de vidro, com seção transversal circular e perfil cônico, com capacidade volumétrica de 2,059 $\mathrm{m}^{3}$ e área superficial de 2,086 $\mathrm{m}^{2}$ (Figura 1). Na Figura 1A, detalha-se o sistema de pesagem hidráulica sob a placa metálica em substituição aos perfis em "U”. Nas Figuras 1B e 1C, apresentam-se o lisímetro montado, bem como o esquema da estrutura de contenção. 


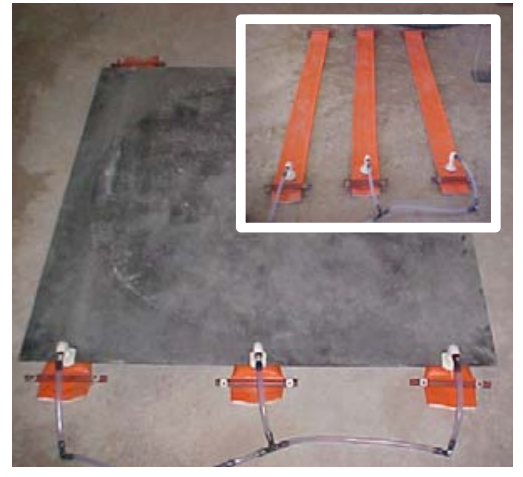

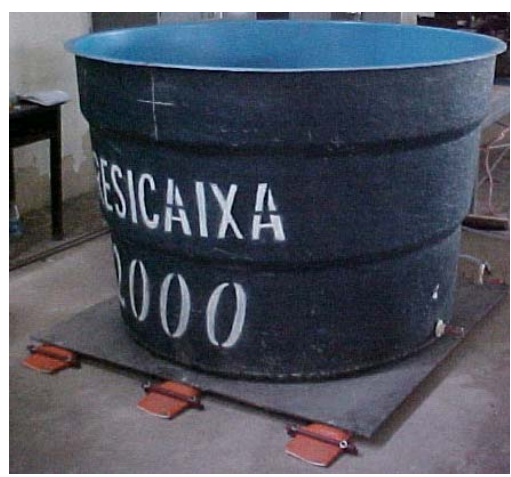

B

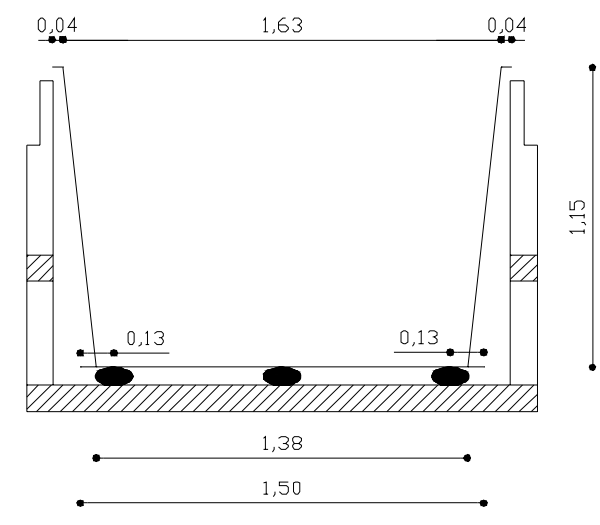

C

FIGURA 1. Configuração do lisímetro, destacando: (A) placa metálica e travesseiros, (B) lisímetro montado, (C) estrutura e detalhe de tanque lisimétrico (medidas em metros). Lysimeter configuration, highlighting: (A) metallic plate and hydraulic pillows, (B) mounted lysimeter, (C) structure and lysimeter tank detail (in meters).

Foram realizadas modificações estruturais em relação aos lisímetros utilizados por FREITAS (1994), COSTA (1999) e SILVA et al. (2003) para diminuir os custos. Para tanto, aboliu-se o acesso subterrâneo ao sistema de pesagem hidráulica e utilizou-se estrutura de contenção mais simples e compacta. Essas modificações foram realizadas, dada a elevada robustez do lisímetro, tornando-se desnecessária a manutenção, como nos lisímetros de balança mecânica ou eletrônica. Outra modificação foi relativa à simplificação no sistema de pesagem, no qual se utilizou placa metálica ao invés de perfis em "U”, sendo os travesseiros hidráulicos dispostos paralelamente entre si. Informações construtivas mais detalhadas podem ser obtidas em SANTOS (2004).

Realizou-se série de três testes de calibração. Cada ensaio de calibração consistiu em carregamento progressivo de massas equivalentes a lâminas de água de $1 \mathrm{~mm}(2,086 \mathrm{~kg})$, seguido de descarregamento, com amplitude de $50 \mathrm{~mm}$ de lâmina de água $(104,3 \mathrm{~kg})$, sendo as leituras efetuadas em manômetro de mercúrio e água graduada em milímetros. A resolução das leituras foi ampliada pela inclinação do tubo manométrico em ângulo de $30^{\circ} \mathrm{com}$ a horizontal. Esse artifício propiciou ampliação da leitura em duas vezes, pois uma variação de cota (c) do fluido, devido à variação de massa do lisímetro, é associada à variação de deslocamento (d) duas vezes maior no tubo manométrico $\left(\operatorname{sen} 30^{\circ}=0,5\right)$, conforme Figura 2 .

Foram realizadas calibrações variando o volume de fluido nos travesseiros hidráulicos, visando a avaliar a flutuação do coeficiente de calibração. Para tanto, procederam-se retiradas sucessivas de volume de $1 \mathrm{~L}$ dos travesseiros hidráulicos, partindo-se de um volume inicial armazenado de $25 \mathrm{~L}$, até um volume final de $15 \mathrm{~L}$. Outro teste foi a calibração em quadrantes para verificar a estabilidade do tanque sobre o sistema de pesagem hidráulica, sob diferentes posições de concentração de carga. O teste consistiu em carregamento seguido de descarregamento, em etapa única, em cada quadrante por vez, com massa equivalente a uma lâmina de água de $40 \mathrm{~mm}$ $(83,44 \mathrm{~kg})$. 


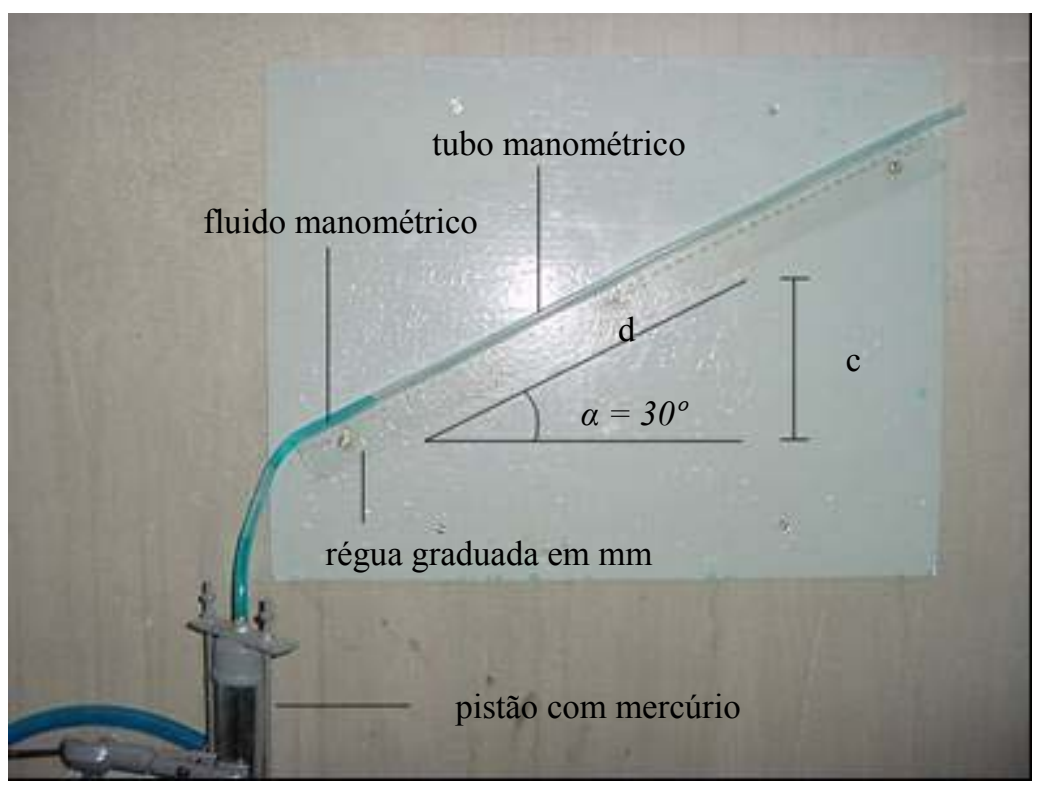

FIGURA 2. Disposição inclinada do tubo manométrico para ampliação da resolução da leitura. c altura do fluido manométrico; d - deslocamento do fluido manométrico. Inclined position of the manometer tube for magnifying reading resolution. c - fluid height; $d$ - fluid displacement.

A área de 1,5 ha de bordadura do lisímetro foi cultivada com "capim-pangola" (Digitaria decumbens Stent.), espécie nativa e abundante na região do vale aluvial, sendo o mesmo mantido com altura máxima de $12 \mathrm{~cm}$. Adotou-se essa mesma planta como grama de referência no lisímetro para medir a evapotranspiração, a qual foi calculada utilizando o princípio da conservação de massas aplicado ao balanço hídrico, com freqüência de irrigação diária. A evapotranspiração pode ser expressa como:

$$
\mathrm{ETo}=\frac{\Delta \mathrm{L}}{\mathrm{f}}+\mathrm{P}-\mathrm{D}-\mathrm{G}
$$

em que,

ETo - evapotranspiração de referência, $\mathrm{mm} \mathrm{dia}^{-1}$;

$\Delta \mathrm{L}$ - variação de leitura, $\mathrm{mm} \mathrm{dia}^{-1}$;

f - coeficiente de calibração para conversão de leitura manométrica $(\mathrm{mm})$ em lâmina $(\mathrm{mm})$;

$\mathrm{P}$ - lâmina precipitada, $\mathrm{mm} \mathrm{dia}^{-1}$;

$\mathrm{D}$ - lâmina drenada, $\mathrm{mm} \operatorname{dia}^{-1}$, e

$\mathrm{G}$ - lâmina de água $\left(\mathrm{mm} \mathrm{dia}^{-1}\right)$ equivalente à massa da grama por ocasião de corte, igual à massa de grama cortada $(\mathrm{kg})$ divida por 2,086, que corresponde à área superficial do lisímetro $\left(\mathrm{m}^{2}\right)$.

$\mathrm{O}$ fator $\mathrm{f}$ também foi adotado na conversão do volume drenado em lâmina drenada. $\mathrm{O}$ volume de água para restabelecimento da capacidade de campo foi calculado com base na variação de massa devido às perdas por drenagem $(\mathrm{Vd})$ e na evapotranspiração para um período de 24 horas, avaliada no manômetro e correspondente à variação de leitura $\Delta \mathrm{L}$. Tem-se, então:

$$
\mathrm{Va}=(\Delta \mathrm{Lr})-\mathrm{Vd}
$$

sendo,

$\mathrm{Va}$ - volume de água para restabelecimento da capacidade de campo, L;

$\mathrm{Vd}$ - volume drenado, L;

$\mathrm{r}$ - coeficiente de conversão de mm de leitura em L de água, e

$\Delta \mathrm{L}$ - variação de leitura no manômetro, $\mathrm{mm}$.

em que, 


$$
\Delta \mathrm{L}=\mathrm{Lf}-\mathrm{Li}
$$

sendo,

Lf e Li - leituras final e inicial, respectivamente.

Tomando o método do lisímetro como padrão, avaliou-se o desempenho dos seguintes métodos: Penman-Monteith (PEREIRA et al., 1997; VESCOVE \& TURCO, 2005), Jensen-Haise (PEREIRA et al., 1997, SILVA et al., 2005), Blaney-Criddle-FAO (DOORENBOS \& PRUITT, 1977; CONCEIÇÃO, 2003), Tanque Classe A-FAO (DOORENBOS \& PRUITT, 1977; SILVA et al., 2005) e tanque de evaporação simplificado. Utilizou-se valor de coeficiente de tanque (Kp) para o Tanque Classe A-FAO de 0,75 (DOORENBOS \& PRUITT, 1977). O tanque simplificado foi construído com tambor metálico de $200 \mathrm{~L}$, cortado transversalmente ao meio, com dimensões de $0,435 \mathrm{~m}$ de altura e $0,57 \mathrm{~m}$ de diâmetro, sendo a leitura realizada diretamente em um paquímetro acoplado a um poço tranqüilizador.

O desempenho dos modelos e do tanque simplificado foi avaliado considerando os coeficientes de regressão (linear e angular), o coeficiente de correlação (r), o erro-padrão da estimativa (EPE), o índice de concordância (d) de Willmott e o índice de desempenho (c) (CAMARGO \& SENTELHAS,1997).

Complementarmente, analisou-se o tamanho da amostra de dados de evapotranspiração de referência quanto à suficiência amostral, com vistas à validação dos dados de campo como representativos para os testes comparativos entre os métodos. MEUNIER et al. (2002) ressaltam que, mediante análise de suficiência amostral, as inferências em amostras de tamanho " $n$ " são suficientes em nível de probabilidade e erro amostral preestabelecidos, podendo ser expressa por:

$$
\mathrm{n}=\frac{\mathrm{t}_{\alpha / 2}^{2} \quad \mathrm{CV}^{2}}{\mathrm{E}^{2}}
$$

em que,

$\mathrm{n}$ - tamanho da amostra representativa da população;

$\mathrm{t}_{\alpha / 2}$ - valor do teste de $\mathrm{t}$ em nível de significância de $\alpha / 2$;

CV - coeficiente de variação (\%), e

E - erro de amostragem (\%).

Adotou-se erro de amostragem de 10\%, conforme recomendado e utilizado por PESSOA (2006), em trabalho de campo na mesma área, a 10\% de significância. Os processos amostrais são função do limite de confiança, no qual a média amostral pode variar numa amplitude de $t_{\alpha / 2} s_{x}^{-}$, dada por:

$$
\mathrm{s}_{\mathrm{x}}=\frac{\mathrm{s}}{\sqrt{\overline{\mathrm{n}}}}
$$

em que,

$\mathrm{s}_{\mathrm{x}}$ - erro-padrão da média;

s - desvio-padrão, e

$\overline{\mathrm{n}}$ - tamanho da amostra-piloto.

Pode-se calcular o erro da amostra-piloto segundo a expressão (SILVA et al.,1997):

$$
\operatorname{EA}(\%)=\left(\frac{\mathrm{t}_{\alpha / 2} \mathrm{~s}_{\mathrm{x}}}{\overline{\mathrm{x}}}\right) 100
$$

em que, 
EA - erro da amostra-piloto;

$\mathrm{s}_{\mathrm{x}}$ - erro-padrão da média;

$\mathrm{t}_{\alpha / 2}-$ valor do teste de $\mathrm{t}$ a um nível de significância $\alpha / 2, \mathrm{e}$

$\overline{\mathrm{x}}$ - média.

A suficiência amostral é atingida quando o erro da amostra-piloto é igual ou menor que o erro de amostragem adotado $(\mathrm{EA} \leq \mathrm{E})$.

\section{RESULTADOS E DISCUSSÃO}

Em relação à concepção de projeto, as modificações no sistema de pesagem trouxeram melhorias ao aparelho. A substituição de perfis em "U", usados por FREITAS (1994), COSTA (1999) e SILVA et al. (2003), por chapa metálica plana (Figura 1A), eliminou paralisações esporádicas do sistema e possibilitou contato entre a chapa e os travesseiros hidráulicos mais bem distribuído.

As calibrações produziram respostas lineares, com coeficientes de correlação superiores a 0,9993, e com histerese desprezível para todos os níveis de volume de fluido dos travesseiros avaliados (Figura 3).

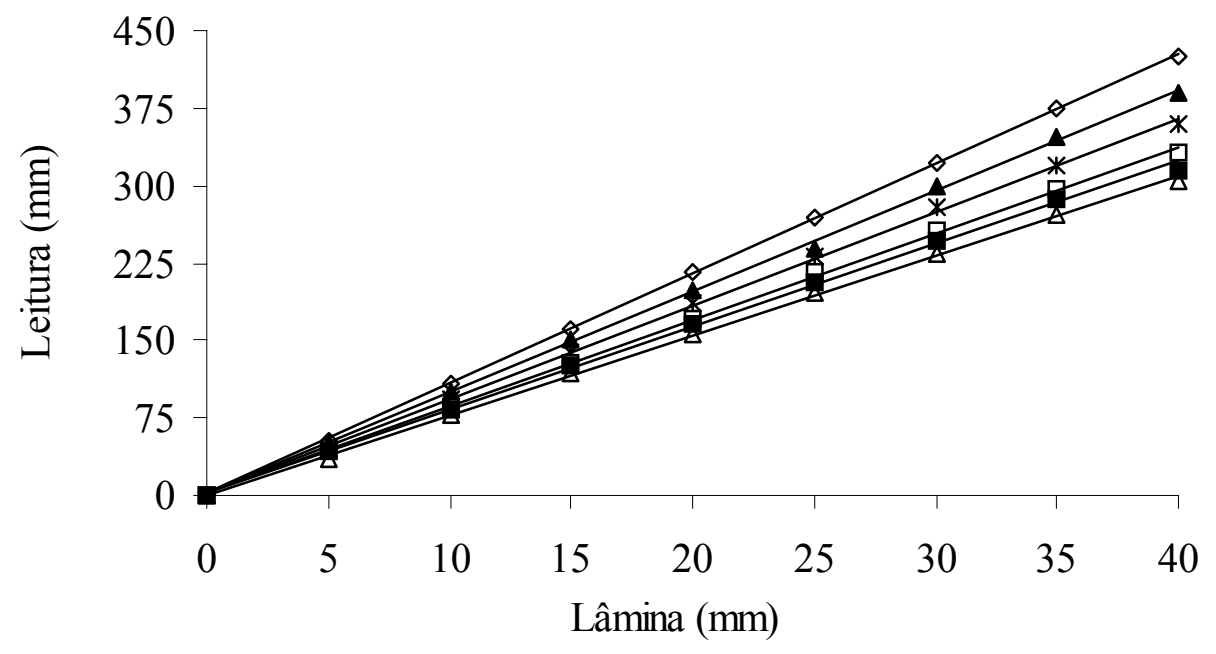
$\diamond 25 ; 0,9999$
\ 23;0,9994
* 21;0,9995
口 19; 0,9995
- $17 ; 0,9988$
$\Delta 15 ; 0,9994$

FIGURA 3. Resultados da calibração do lisímetro para diferentes volumes no travesseiro (L). Legenda: volume de fluido nos travesseiros; coeficiente de determinação. Lysimeter calibration results for different volumes in the hydraulic pillow. Legend: fluid volume in the pillows; determination coefficient.

Verifica-se que quanto maior o volume de fluido nos travesseiros do lisímetro, maior foi a sua sensibilidade, produzindo medidas de ETo de maior resolução. Com as reduções do volume nos travesseiros, a sensibilidade decresce e tende a um valor de precisão estável.

Na Tabela 1, são apresentados os coeficientes de calibração sob diferentes níveis de volume de fluido nos travesseiros. À medida que os travesseiros são deflacionados, o coeficiente de calibração (f) diminui. Isso ocorre porque a área de contato chapa-travesseiro aumenta para mesma força de aplicação, o que incorre em redução de pressão. Assim, o mesmo incremento ou decremento de carga do tanque provoca variação de pressão nos travesseiros inversamente proporcional à área de contato. 
TABELA 1. Resultados dos ensaios de calibração com retiradas progressivas de volume $(1 \mathrm{~L})$ dos travesseiros hidráulicos, sob carregamento simetricamente distribuído. Calibration results for the tests with gradual volume withdrawals $\left(\begin{array}{ll}1 & L\end{array}\right)$ of the hydraulic pillows, under symmetrical loading.

\begin{tabular}{cccccccccccc}
\hline $\begin{array}{c}\text { Volume de } \\
\text { fluido }(\mathrm{L})\end{array}$ & 25 & 24 & 23 & 22 & 21 & 20 & 19 & 18 & 17 & 16 & 15 \\
\hline $\mathrm{f}\left(\mathrm{mm} \mathrm{mm}^{-1}\right)^{*}$ & 10,54 & 9,97 & 9,48 & 9,13 & 8,90 & 8,61 & 8,22 & 7,69 & 7,68 & 7,78 & 7,46 \\
\hline Precisão $(\mathrm{mm})$ & 0,095 & 0,100 & 0,105 & 0,109 & 0,112 & 0,116 & 0,121 & 0,130 & 0,130 & 0,129 & 0,134 \\
\hline
\end{tabular}

*coeficiente de calibração

Os erros posicionais obtidos são apresentados na Tabela 2 e indicam que o lisímetro pode operar, sob condições de equilíbrio estável, em todos os volumes de fluidos testados nos travesseiros (Tabela 2). FREITAS (1994) e COSTA (1999) recomendam como limite tolerável 10\% de erro posicional. Os referidos autores obtiveram $4 \%$ e 3,55\% de erro posicional, respectivamente, em lisímetros de pesagem hidráulica, enquanto SILVA et al. (2003) obtiveram 2,11\%.

TABELA 2. Erro médio posicional do coeficiente de calibração $\mathrm{f}\left(\mathrm{mm} \mathrm{mm}^{-1}\right)$, sob diferentes níveis de volumes de fluidos dos travesseiros e carregamentos simétrico e assimétrico do lisímetro. Positional average error of the calibration coefficient $\mathbf{f}\left(\mathrm{mm} \mathrm{mm}^{-1}\right)$, under different levels of fluid volume in the pillows and symmetrical and antisymmetrical loading.

\begin{tabular}{cccccc}
\hline \multirow{2}{*}{ Quadrante } & \multicolumn{5}{c}{ Volume de Fluido nos Travesseiros (L) } \\
\cline { 2 - 5 } & 28 & 25 & 22 & 19 & 16 \\
\hline Primeiro & 11,67 & 10,11 & 8,95 & 7,94 & 7,47 \\
Segundo & 11,91 & 9,88 & 8,80 & 7,86 & 7,41 \\
Terceiro & 12,29 & 9,98 & 8,93 & 7,71 & 7,64 \\
Quarto & 12,28 & 10,15 & 9,14 & 7,99 & 7,73 \\
f médio (assimétrico) & 12,04 & 10,03 & 8,96 & 7,88 & 7,56 \\
\hline f médio (simétrico) & 11,93 & 10,44 & 8,90 & 7,69 & 7,46 \\
\hline Erro posicional (\%) & 0,92 & $-3,93$ & 0,67 & 2,47 & 1,34 \\
\hline
\end{tabular}

A ocorrência de eventos pluviométricos reduziu a taxa evapotranspirativa acumulada no final do período analisado, mais notadamente para o Tanque Classe " $A$ ". Uma das limitações do uso dos tanques evaporimétricos é a ocorrência de eventos pluviométricos. Nos dias de chuva, o manejo dos tanques foi prejudicado devido a erros de leituras de evaporação. Outro fator, de cunho estrutural e normativo, refere-se à faixa de operação da escala dos tanques, cuja amplitude é limitada aos $50 \mathrm{~mm}$ de altura útil para leitura (OMETTO, 1981; SANTIAGO et al., 2004).

Quanto ao tanque simplificado, devido à falta de Kp determinado experimentalmente, esse foi confrontado com o Tanque Classe A-FAO e com o método-padrão, levando-se em consideração apenas a evaporação. A evaporação acumulada foi de $289,72 \mathrm{~mm}$, enquanto no Tanque Classe AFAO obtiveram-se $236,66 \mathrm{~mm}$, o que representa cerca de $22 \%$ a mais para o tanque simplificado. Essa variação em relação ao Tanque Classe A-FAO pode ser atribuída às particularidades geométricas e ao material de constituição dos tanques, que conferem diferenças quanto à área superficial de exposição e quanto à absorção da radiação solar. SENTELHAS \& FOLEGATTI (2003) ressaltam a necessidade de avaliar e de calibrar os valores de Kp sugeridos na literatura, bem como produzi-los para as condições climáticas locais. Para a correção da evaporação do tanque simplificado, adotou-se, para o período utilizado, o valor de 0,60 em relação ao lisímetro, ou a relação $\mathrm{Kp}_{\mathrm{s}}=0,81 \mathrm{Kp}$, em que $\mathrm{Kp}$ é o coeficiente do Tanque Classe $\mathrm{A}$, e $\mathrm{Kp}_{\mathrm{s}}$ é o coeficiente do tanque simplificado.

Adotando-se o volume de fluido nos travesseiros de $16 \mathrm{~L}$, e o valor do coeficiente de calibração conforme a Tabela $1\left(7,78 \mathrm{~mm} \mathrm{~mm}^{-1}\right)$, o lisímetro acumulou 173,96 mm de ETo, com 
média de 5,80 $\mathrm{mm} \mathrm{dia}^{-1}$. A ETo acumulada avaliada pelo método de Penman-Monteith superestimou o método-padrão (Tabela 3). SILVA et al. (2003), sob condições do Semi-Árido nordestino, obtiveram resultados semelhantes aos do presente estudo, com coeficiente de correlação entre medidas lisimétricas e o modelo de Penman-Monteith de 0,85 . Os coeficientes de desempenho dos demais métodos estão também apresentados na Tabela 3.

TABELA 3. Parâmetros de avaliação dos métodos estimativa da ETo em 30 observações diárias, segundo o índice "c", tomando-se como padrão o método do lisímetro. Evaluation parameters of the ETo estimative methods in 30 daily observations, according to index "c", considering the lysimeter method as the standard.

\begin{tabular}{cccccccccc}
\hline Método & EAC & DR & EPE & \multicolumn{1}{c}{ a } & \multicolumn{1}{c}{ b } & \multicolumn{1}{c}{ r } & \multicolumn{1}{c}{ d } & \multicolumn{1}{c}{ c } & Desempenho \\
\hline Tanque Classe A FAO & 175,96 & 0,67 & 1,05 & 0,97 & 0,83 & 0,86 & 0,93 & 0,80 & Muito bom \\
Tanque simplificado* & 173,96 & 0,00 & 1,20 & 1,61 & 0,72 & 0,86 & 0,92 & 0,79 & Muito bom \\
Blaney-Criddle-FAO & 175,79 & 0,96 & 1,08 & 0,01 & 0,99 & 0,84 & 0,91 & 0,76 & Muito bom \\
Penman-Monteith & 190,53 & 8,70 & 1,15 & $-0,45$ & 0,98 & 0,86 & 0,90 & 0,77 & Muito bom \\
Jensen-Haise & 201,05 & 14,22 & 1,38 & 0,13 & 0,85 & 0,86 & 0,88 & 0,76 & Muito bom \\
tanque simplificado & 217,29 & 22,74 & 2,15 & 1,61 & 0,58 & 0,86 & 0,81 & 0,76 & Muito bom \\
\hline
\end{tabular}

EAC - evapotranspiração acumulada (mm); DR - desvio relativo à EAC do método do lisímetro (\%); EPE - erro-padrão da estimativa $\left(\mathrm{mm} \mathrm{dia}^{-1}\right) ; a$ e $b$ - coeficientes linear e angular da regressão; $\mathrm{r}$ - coeficiente de correlação; $\mathrm{d}$ - índice de concordância, c - índice de desempenho. *considerando o coeficiente de tanque de 0,60 .

PEREIRA et al. (1997) comentam que, embora o método de Blaney-Criddle-FAO possa ser adotado para a estimativa em escalas menores que 30 dias, a sua representatividade é questionável, visto que o método foi desenvolvido considerando dados médios mensais. Neste estudo, o método de Blaney-Criddle-FAO apresentou estimativas de ETo diária bem correlacionados $(\mathrm{r}=0,84)$ e concordantes $(d=0,91)$. O modelo de Jensen-Haise apresentou elevada concordância $(d=0,88)$; no entanto, produziu sobrestimativa de 14,22\% em relação ao lisímetro. Esse modelo é simples, necessitando apenas da temperatura média diária e da radiação solar, constituindo-se em método de fácil aplicação e de resultados razoáveis à condição local. SILVA et al. (2003) relatam que esse método foi pouco estudado no Brasil e o aplicaram sob condições climáticas semelhantes, registrando elevada consistência dos dados, tanto em correlação $(0,81)$ quanto em concordância $(0,94)$.

O método do Tanque Classe " $A$ " - FAO apresentou desempenho superior a todos os demais métodos avaliados, na maioria dos quesitos considerados, com valores médios diários muito próximos de $5,84 \mathrm{~mm} \mathrm{dia}{ }^{-1}$ contra $5,80 \mathrm{~mm} \mathrm{dia}^{-1}$ do lisímetro, ao contrário do observado por OMETTO (1981), que afirma ser o referido método tendencioso à sobrestimativa, e por SILVA et al. (2003), que registraram subestimativa e correlação com medidas lisimétricas de 0,67.

Embora se tenha trabalhado com uma série de dados relativamente curta, os dados utilizados nos testes comparativos foram suficientes em $10 \%$ de significância pelo teste $t$, e conduziram a erro de amostra-piloto (EA) inferior ao erro de amostragem (E) estabelecido, para dados de campo, de $10 \%$, conforme PESSOA (2006), indicando, assim, que os dados são provenientes de uma série de observações suficientemente grande para ser representativa (Tabela 4). O tamanho amostral disponível foi de 30, com exceção dos métodos do Tanque Classe A-FAO, do tanque simplificado e do lisímetro, cujo tamanho foi reduzido para 26. A retirada de quatro observações teve o objetivo de homogeneizar a amostra e isolar os dias chuvosos, dada a interferência desses na qualidade dos dados medidos com os referidos métodos. 
TABELA 4. Parâmetros de avaliação quanto à representatividade dos dados de evapotranspiração de referência. Evaluation parameters for the data representation of reference evapotranspiration.

\begin{tabular}{crrrrrr}
\hline Método & Pn-Mt & TCA & TS* & Bn-Cd & Jn-Hs & \multicolumn{1}{c}{ Lis* } \\
\hline CV (\%) & 27,71 & 17,36 & 25,10 & 29,05 & 30,70 & 18,78 \\
n & 22,19 & 8,71 & 18,20 & 24,39 & 27,24 & 10,19 \\
EA (\%) & 8,60 & 5,39 & 7,79 & 9,02 & 9,53 & 5,82 \\
\hline
\end{tabular}

CV - coeficiente de variação; $n$ - tamanho da amostra representativa da população; $E A$ - erro da amostra-piloto. *Amostra de tamanho 26; Pn-Mt - Penman-Monteith; TCA - Tanque Classe "A" - FAO; TS - tanque simplificado; BnCd - Blaney-Criddle FAO; Jn-Hs - Jensen-Haise; Lis - lisímetro de pesagem hidráulica.

\section{CONCLUSÕES}

A concepção simplificada de projeto do lisímetro não interferiu em sua funcionalidade, constituindo-se em alternativa de baixo custo para medições lisimétricas de demanda diária.

O lisímetro apresentou equilíbrio estável e leituras de ETo consistentes em todos os ensaios de calibração, com respostas lineares e histerese desprezível.

As medidas de ETo do lisímetro foram bem correlacionadas com as estimativas dos métodos indiretos, sendo o Tanque Classe A o que apresentou o melhor desempenho entre os métodos avaliados, e o tanque simplificado constituiu-se em equipamento promissor para a agricultura familiar irrigada.

\section{AGRADECIMENTOS}

À CAPES e ao REHISA-FINEP, pelo apoio financeiro e logístico na realização deste trabalho. Os autores agradecem aos revisores do artigo, que muito contribuíram para o seu aprimoramento.

\section{REFERÊNCIAS}

ABOUKHALED, A.; ALFARO, A.; SMITH, M. Lysimeters. Rome: FAO, 1982. 68 p. (Irrigation and Drainage Paper, 39).

CAMARGO, A.P.; SENTELHAS, P.C. Avaliação do desempenho de diferentes métodos de estimativa da evapotranspiração potencial no Estado de São Paulo. Revista Brasileira de Agrometeorologia, Santa Maria, v.5, n.1, p.89-97, 1997.

CISAGRO. COMPANHIA INTEGRADA DE SERVIÇOS AGROPECUÁRIOS. Projeto de irrigação da Fazenda Nossa Senhora do Rosário. Pesqueira, 1990. 120 p.

CONCEIÇÃO, M.A.F. Estimativa da evapotranspiração de referência com base na temperatura do ar para as condições do Baixo Rio Grande - SP. Revista Brasileira de Agrometeorologia, Santa Maria, v.11, n.2, p.229-36, 2003.

COSTA, M.S.V. Determinação da evapotranspiração de referência por evapotranspirômetro de pesagem hidráulica com arranjo de "pistão de mercúrio". 1999. 85 f. Dissertação (Mestrado em Ciência do Solo) - Universidade Federal Rural de Pernambuco, Recife, 1999.

DOORENBOS, J.; PRUITT, W.O. Guidelines for predicting crop water requirements. Rome: FAO, 1977. 179 p. (Irrigation and Drainage Paper, 24).

FREITAS, J.A.G. Evapotranspirômetro por sensor de carga hidráulica: construção, calibração e testes. 1994. 122 f. Dissertação (Mestrado em Ciência do Solo) - Universidade Federal Rural de Pernambuco, Recife, 1994. 
LUNARDI, M.A.; LUNARDI, D.M.C.; CAVAGUTI, N. Comparação entre medidas evapotranspirométricas e metodologia da FAO, na determinação da evapotranspiração de referência. Irriga, Botucatu, v.4, n.1, p.52-66, 1999.

MENDONÇA, J.C.; SOUSA, E.F.; BERNARDO, S.; DIAS, G.P.; GRIPPA, S. Comparação entre métodos de estimativa da evapotranspiração de referência (ETo) na região Norte Fluminense, RJ. Revista Brasileira de Engenharia Agrícola e Ambiental, Campina grande, v.7, n.2, p.275-9, 2003.

MEUNIER, I.M.J.; SILVA, J.A.A.; FERREIRA, R.L.C. Inventário florestal: programa de estudo. Recife: UFRPE, 2002. 189 p.

MIRANDA, F.R.; YODER, R.E.; SOUSA, F. Instalação e calibração de um lisímetro de pesagem no projeto de irrigação Curu-Paraipaba, CE. Revista Brasileira de Engenharia Agrícola e Ambiental, Campina Grande, v.3, n.1, p.107-10, 1999.

OMETTO, J.C. Bioclimatologia vegetal. São Paulo: Agronômica Ceres, 1981. 440 p.

PEREIRA, A.R.; VILLA NOVA, N.A.; SEDIYAMA, G.C. Evapo(trans)piração. Piracicaba: FEALQ, 1997. 183 p.

PESSOA, A.L. Uma abordagem baeysiana para o estudo estatístico e geoestatístico de salinidade do solo utilizando sensor de indução eletromagnética. 2006. 112 f. Dissertação (Mestrado em Biometria) - Universidade Federal Rural de Pernambuco, Recife, 2006.

SANTIAGO, F.S.; MONTENEGRO, A.A.A.; MONTENEGRO, S.M.G.L. Avaliação de parâmetros hidráulicos e manejo da irrigação por microaspersão em área de assentamento. Engenharia Agrícola, Jaboticabal, v.24, n.3, p.632-43, 2004.

SANTOS, F.X. Lisímetro de pesagem hidráulica e evapotranspiração de referência por diferentes métodos no agreste pernambucano. 2004. 77 f. Dissertação (Mestrado em Ciência do Solo) Universidade Federal Rural de Pernambuco, Recife, 2004.

SCHNEIDER, A.D.; HOWELL, T.A.; MOUSTAFA, A.T.A.; EVETT, S.R.; ABOU-ZEID, W. A simplified weighing lysimeter for monolithic or reconstructed soils. American Society of Agricultural Engineers, St. Joseph, v.14, n.3, p.267-73, 1998.

SENTELHAS, P.C.; FOLEGATTI, M.V. Class A pan coefficients (Kp) to estimate daily reference evapotranspiration. Revista Brasileira de Engenharia Agrícola e Ambiental, Campina Grande, v.7, n.1, p.111-15, 2003.

SILVA, J.A.A.; CAMPOS, A.L.; VEIGA, A.F.S.L.; MOREIRA, A.F.C.; MARQUES, E.J. Estimativa da suficiência amostral para avaliar intensidade de infestação da Diatraea ssp, em cana-de-açúcar. Pesquisa Agropecuária Brasileira, Brasília, v.32, n.10, p.1003-7, 1997

SILVA, T.J.A. Evapotranspiração e coeficiente de cultivo de maracujazeiro determinados pelo método do balanço de radiação e lisimetria de pesagem hidráulica. 2005. 99 f. Tese (Doutorado em Irrigação e Drenagem) - Escola Superior de Agricultura "Luiz de Queiroz", Piracicaba, 2005.

SILVA, T.J.A.; MONTENEGRO, A.A.; RODRIGUES, J.J.V.; BONFIM, E.M.S. Aplicação de lisímetro de pesagem hidráulica na determinação da evapotranspiração de referência, em Petrolina PE. Engenharia Agrícola, Jaboticabal, v.23, n.3, p.511-20, 2003.

SILVA, V.P.R.; BELO FILHO, A.F.; SILVA, B.B.; CAMPOS, J.H.B. Desenvolvimento de um sistema de estimativa da evapotranspiração de referência. Revista Brasileira de Engenharia Agrícola e Ambiental, Campina Grande, v.9, n.4, p.547-53, 2005

VESCOVE, H.V.; TURCO, J.E.P. Comparação de três métodos de estimativa da evapotranspiração de referência para a região de Araraquara - SP. Engenharia Agrícola, Jaboticabal, v.25, n.3, p.71321, 2005. 